\title{
Optical and magneto-optical far-infrared properties of bilayer graphene
}

\author{
D. S. L. Abergel and Vladimir I. Fal'ko \\ Physics Department, Lancaster University, Lancaster, LA1 4YB, UK
}

\begin{abstract}
We analyze the spectroscopic features of bilayer graphene determined by the formation of pairs of low-energy and split bands in this material. We show that the inter-Landau-level absorption spectrum in bilayer graphene at high magnetic field is much denser in the far-infrared range than that in monolayer material, and that the polarization dependence of its lowest-energy peak can be used to test the form of the bilayer ground state in the quantum Hall-effect regime.
\end{abstract}

Monolayer and bilayer graphenes $\frac{1,2.3}{4.5}$ are gapless twodimensional (2D) semiconductors $\frac{4.5}{4}$. When used in transistor-type devices, the density and type ( $n$ or $p)$ of carriers in them can be controlled using the underlying gate ${ }^{\underline{1}}$, which has been exploited in the recent studies of the quantum Hall effect (QHE) in such structures. The sequencing of QHE plateaus in graphene $e^{1,2,3}$ revealed the peculiar properties of the charge carriers in this material: Dirac-type chiral electrons with Berry phase $\pi$ in monolayers 4,6 , and the Berry phase $2 \pi$ chiral quasiparticles ${ }^{5}$ with doubled degeneracy of the zeroenergy Landau level (LL) in bilayers.

The electromagnetic (EM) field absorption in graphene at zero magnetic field has already been studied $7,8,9,10$. While the dc conductivity of monolayer graphene increases linearly with the carrier density $\stackrel{111}{1}$, the real part of its high-frequency conductivity $\underline{\underline{7} .9}$ is independent of the electron density in a wide spectral range above the threshold $\hbar \omega>2\left|\epsilon_{\mathrm{F}}\right|$, which determines a featureless absorption coefficient $g_{1}=\pi e^{2} / \hbar c$. In contrast, the bilayer absorption coefficient,

$$
g_{2}=\left(2 \pi e^{2} / \hbar c\right) f_{2}(\omega)
$$

reflects the presence and dispersion of two pairs of bands in this material $5^{5,8,12,13}$. In this paper, we analyse farinfrared (FIR) magneto-optical properties due to the inter-LL $\frac{5,14,15}{1}$ transitions in bilayer graphene and compare them to those in the monolayer material. The lowenergy part of the absorption spectrum in a bilayer subjected to a strong perpendicular magnetic field is much denser than that of a monolayer, reflecting the parabolic dispersion of its low-energy bands, with the lowest FIR absorption peak formed by transitions involving one of two degenerate LLs at zero energy. Although the highenergy part of the bilayer absorption spectrum is not sensitive to the polarisation of FIR irradiation, below we show that its lowest peak may be strongly polarised reflecting the occupancy of the degerate LLs in a bilayer determined by the form of its ground state in the QHE regime.

The electronic Fermi line in graphene surrounds the corners $\frac{16}{} \mathbf{K}_{ \pm}$of the hexagonal Brillouin zone ${ }^{4}$ (where we set $\epsilon=0$ ). In monolayer graphene, quasiparticles near the centres of valleys $\mathbf{K}_{ \pm}$can be described by 4 component Bloch functions $\psi=\left[\phi_{\mathbf{K}_{+} A}, \phi_{\mathbf{K}_{+} B}, \phi_{\mathbf{K}_{-} B}\right.$, $\left.\phi_{\mathbf{K}_{-} A}\right]$ and the Hamiltonian $\hat{H}_{1}=v \Pi_{z} \otimes \boldsymbol{\sigma} \cdot \mathbf{p}$, where $\boldsymbol{\sigma}=\left(\sigma_{x}, \sigma_{y}\right)$ are Pauli matrices acting in the space of electronic amplitudes on the two crystalline sublattices $(A$ and $B)$ and $\Pi_{z}$ is the diagonal Pauli matrix in the valley space. Momentum $\mathbf{p}=-i \hbar \nabla-\frac{e}{c} \mathbf{A}$ is calculated with respect to the center of the corresponding valley and $\nabla \times \mathbf{A}=B \mathbf{l}_{z}$.

Bilayer graphene is composed of two coupled monolayers (with sublattices $A, B$ and $\tilde{A}, \tilde{B}$ in the bottom and top layers respectively) arranged according to Bernal stacking $\stackrel{4}{4}$ : sites $B$ of the honeycomb lattice in the bottom layer lie below $\tilde{A}$ of the top layer. It also has a hexagonal Brillouin zone with two inequivalent valleys, but carries twice the number of electronic dispersion branches. The latter can be found using the nearest-neighbour hopping Hamiltonian ${ }^{\underline{5}}$ acting in the space of sublattice states $\left[\phi_{\mathbf{K}_{+} A}, \phi_{\mathbf{K}_{+} \tilde{B}}, \phi_{\mathbf{K}_{+} \tilde{A}}, \phi_{\mathbf{K}_{+} B} ; \phi_{\mathbf{K}_{-} \tilde{B}}, \phi_{\mathbf{K}_{-} A}, \phi_{\mathbf{K}_{-} B}\right.$, $\left.\phi_{\mathbf{K}_{-} \tilde{A}}\right]$,

$$
\hat{\mathcal{H}}_{2}=\left(\begin{array}{cc}
v_{3} \Pi_{z} \otimes \boldsymbol{\sigma}^{\mathrm{t}} \cdot \mathbf{p} & v \Pi_{z} \otimes \boldsymbol{\sigma} \cdot \mathbf{p} \\
v \Pi_{z} \otimes \boldsymbol{\sigma} \cdot \mathbf{p} & \gamma_{1} \Pi_{0} \otimes \sigma_{x}
\end{array}\right) .
$$

Here, $v$ is determined by the $A B(\tilde{A} \tilde{B})$ intra-layer hopping, $\gamma_{1}$ is the strongest inter-layer $\tilde{A} B$ hopping element, and $v_{3} \ll v$ is due to a weak direct $A \tilde{B}$ hop, and 't' stands for transposition. Equation (2) determines two types of branches in the electronic spectrum of graphene 5.12 observed in the recent ARPES studies ${ }^{13}$ : Split-bands,

$$
\varepsilon_{s}^{ \pm}= \pm\left(\gamma_{1} / 2\right)\left[\sqrt{1+4 v^{2} p^{2} / \gamma_{1}^{2}}+1\right],
$$

formed by symmetric and antisymmetric states based upon $\tilde{A} B$ sublattices; and two gapless branches, $\varepsilon_{c}^{ \pm}$. For $\epsilon \gg \epsilon_{\mathrm{L}} \equiv \frac{1}{2} \gamma_{1}\left(v_{3} / v\right)^{2}$, the dispersion in gapless branches can be approximated by

$$
\varepsilon_{c}^{ \pm}= \pm\left(\gamma_{1} / 2\right)\left[\sqrt{1+4 v^{2} p^{2} / \gamma_{1}^{2}}-1\right] .
$$

For $|\epsilon|<\frac{1}{4} \gamma_{1}$, gapless bands are formed by states from sublattices $A$ and $\tilde{B}$ described using the 4-component Bloch functions $\chi=\left[\phi_{\mathbf{K}_{+} A}, \phi_{\mathbf{K}_{+} \tilde{B}}, \phi_{\mathbf{K}_{-} \tilde{B}}, \phi_{\mathbf{K}_{-} A}\right]$ and the reduced low-energy Hamiltonian ${ }^{5,17}$,

$$
\begin{gathered}
\hat{H}_{2}=-\frac{1}{2 m_{2}}(\boldsymbol{\sigma} \cdot \mathbf{p}) \sigma_{x}(\boldsymbol{\sigma} \cdot \mathbf{p})+\delta \hat{h}_{w}+\frac{\beta p^{2}}{m_{2}} ; \\
\delta \hat{h}_{w}=v_{3} \Pi_{z} \otimes \boldsymbol{\sigma}^{\mathrm{t}} \cdot \mathbf{p} ; \quad m_{2}=\gamma_{1} / 2 v^{2},|\beta| \ll 1 .
\end{gathered}
$$

Here $m_{2} \approx 0.05 m_{e}$ and the last term in $\hat{H}_{2}$ takes into account the weak $A A$ and $B B$ intra-layer hopping 18 . 
In a $2 \mathrm{D}$ electron gas with conductivity $\sigma(\omega)$ much less than $c / 2 \pi$, absorption of an EM field $\mathbf{E}_{\omega}=\ell E e^{-i \omega t}$ with polarisation $\boldsymbol{\ell}\left(\boldsymbol{\ell}_{\oplus}=\left[\mathbf{l}_{x}-i \mathbf{l}_{y}\right] / \sqrt{2}\right.$ for right- and $\boldsymbol{\ell}_{\ominus}=\left[\mathbf{1}_{x}+i \mathbf{1}_{y}\right] / \sqrt{2}$ for left-hand circularly polarised light,respectively arriving along the direction antiparallel to a magnetic field) can be characterised by the absorption coefficient $g \equiv E_{i} E_{j}^{*} \sigma_{i j}(\omega) / S$ : the ratio between Joule heating and the energy flux $\mathbf{S}=c \mathbf{E} \times \mathbf{H} / 4 \pi=$ $-S \mathbf{l}_{z}$ transported by the EM field. Using the Keldysh technique, we express

$$
g=\frac{8 e^{2}}{c \omega} \Re \int \frac{F d \epsilon}{N} \widehat{\operatorname{Tr}}\left\{\hat{v}_{i} \ell_{i} \hat{G}^{R}(\epsilon) \hat{v}_{j} \ell_{j} \hat{G}^{A}(\epsilon+\omega)\right\},
$$

where $\hat{\mathbf{v}}=\partial_{\mathbf{p}} \hat{H}$ is the velocity operator, $\widehat{T r}$ includes the summation both over the sublattice indices "tr" and over single-particle orbital states, $N$ is the normalisation area of the sample and $F=n_{\mathrm{F}}(\epsilon)-n_{\mathrm{F}}(\epsilon+\omega)$ takes into account the occupancy of the initial and final states. Here we have included spin and valley degeneracy.

For a 2D gas in a zero magnetic field, the electron states are weakly scattered plane waves. Using the plane wave basis and the matrix form of the high energy bilayer Hamiltonian $\hat{\mathcal{H}}_{2}$, we express the retarded/advanced Greens functions of electrons in the bilayer as $\hat{G}^{R / A}(\mathbf{p}, \epsilon)=\left[\epsilon \pm \frac{1}{2} i \hbar \tau^{-1}-\hat{H}_{2}(\mathbf{p})\right]^{-1}$ and $\widehat{\operatorname{Tr}}=\int d^{2} \mathbf{p} \frac{N}{(2 \pi \hbar)^{2}} \mathrm{tr}$, neglecting the renormalisation of the current operator by vertex corrections at $\omega \tau \gg 1$ and the momentum transfer from light (since $v / c \sim 3 \times 10^{-3}$ ). This reproduces the constant absorption coefficient $g_{1}^{\|}=$ $\pi e^{2} / \hbar c$ so that $f_{1}=\frac{1}{2} 7,9$ in monolayer graphene and yields the following expression for the absorption coefficient of bilayer graphene for light polarised in the plane of its sheet 19 :

$$
\begin{gathered}
g_{2}^{\|}=\frac{2 \pi e^{2}}{\hbar c} f_{2}(\Omega), \quad \Omega \equiv \frac{\hbar \omega}{\gamma_{1}}>\frac{2\left|\epsilon_{\mathrm{F}}\right|}{\gamma_{1}}, \\
f_{2}=\frac{\Omega+2}{2(\Omega+1)}+\frac{\theta(\Omega-1)}{\Omega^{2}}+\frac{(\Omega-2) \theta(\Omega-2)}{2(\Omega-1)} .
\end{gathered}
$$

Here $\theta(x<0)=0$ and $\theta(x>0)=1$. This result agrees with the calculation by J. Nilsson et al $\underline{\underline{8}}$ taken in the clean limit and at $T=0$. The frequency dependence $e^{20}$ of the bilayer optical absorption is illustrated in Fig 1. where an additional structure in the vicinity of $\hbar \omega=$ $\gamma_{1}\left(\gamma_{1} \approx 0.4 \mathrm{eV}\right.$ (Refs. $\left.\stackrel{\underline{4}}{\text { and }^{13}}\right)$ is due to the electronhole excitation between the low-energy band $\varepsilon_{c}^{ \pm}$and the split band $\varepsilon_{s}^{ \pm}$. For the higher photon energies, $\hbar \omega \gtrsim$ $2 \gamma_{1}$, the frequency dependence almost saturates at $f \approx$ 1. Over the entire spectral interval shown in Fig 1, the absorption coefficient for the left- and right-handed light are the same, so that Eq. (7) is applicable $\underline{\underline{19}}$ to light linearly polarised in the graphene plane.

High-field FIR magneto-optics of graphene. In a magnetic field, the continuous zero-field spectrum, [Eq. (77)] splits into lines determined by transitions between Landau levels. The LLs can be studied after rewriting $\hat{H}_{1}$

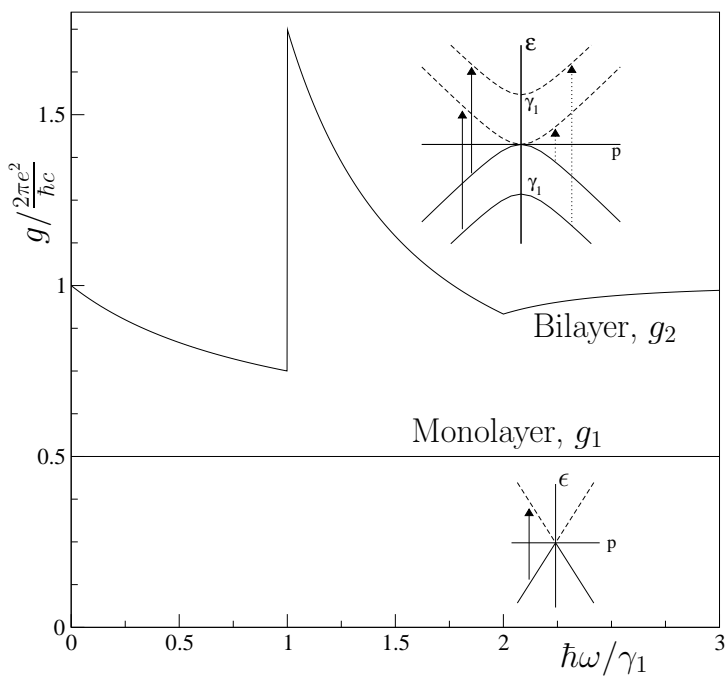

FIG. 1: Absorption coefficient of bilayer and monolayer graphene in the optical range of frequencies. Insets illustrate the quasiparticle dispersion branches in the vicinity of $\epsilon_{F}$ and possible optical transitions.

and $\hat{H}_{2}$ in terms of descending, $\pi=p_{x}+i p_{y}$ and raising, $\pi^{\dagger}=p_{x}-i p_{y}$ operators in the basis of Landau functions $\varphi_{n \geq 0}$, leading to

$$
\boldsymbol{\sigma} \cdot \boldsymbol{p}=\left(\begin{array}{cc}
0 & \pi^{\dagger} \\
\pi & 0
\end{array}\right)
$$

The resulting monolayer spectrum $\frac{14}{4}$ contains 4 -fold degenerate $(2 \times$ spin and $2 \times$ valley index $)$ states: one at $\epsilon_{0}=0$ with $\psi_{0 K_{+}}=\left[\varphi_{0}, 0,0,0\right]$ and $\psi_{0 K_{-}}=$ $\left[0,0, \varphi_{0}, 0\right]$, and pairs of levels $\epsilon_{n \pm}= \pm \hbar v \lambda_{B}^{-1} \sqrt{2 n}$ $\left(\lambda_{B}=\sqrt{\hbar c / e B}\right.$ is the magnetic length) with $\psi_{n \pm, K_{+}}=$ $\frac{1}{\sqrt{2}}\left[\varphi_{n}, \pm i \varphi_{n-1}, 0,0\right]$ and $\psi_{n \pm, K_{-}}=\frac{1}{\sqrt{2}}\left[0,0, \varphi_{n}, \mp i \varphi_{n-1}\right]$. We neglect the electron spin splitting and use the index $\alpha= \pm$ in $\epsilon_{n \alpha}$ and $\psi_{n \alpha, K}$ for the conduction $(+)$ and valence $(-)$ band LLs.

The bilayer spectrum features ${ }^{\frac{5}{4}}$ a group of eight states with $\varepsilon_{0} \approx \varepsilon_{1} \approx 0$ with wave functions $\chi_{0, K_{+}}=$ $\left[\varphi_{0}, 0,0,0\right], \chi_{0, K_{-}}=\left[0,0, \varphi_{0}, 0\right]$ and $\chi_{1, K_{+}}=\left[\varphi_{1}, 0,0,0\right]$, $\chi_{1, K_{-}}=\left[0,0, \varphi_{1}, 0\right](4 \times 2$, due to spin degeneracy), and a ladder of almost equidistant 4-fold degenerate levels $\varepsilon_{n \pm}= \pm \hbar \omega_{c} \sqrt{n(n-1)}(2 \times$ spin and $2 \times$ the valley index) with $\hbar \omega_{c}=\hbar^{2} / m_{2} \lambda_{B}^{2}$ and wave functions $\chi_{n \pm, K_{+}}=$ $\frac{1}{\sqrt{2}}\left[\varphi_{n}, \pm \varphi_{n-2}, 0,0\right], \chi_{n \pm, K_{-}}=\frac{1}{\sqrt{2}}\left[0,0, \varphi_{n}, \pm \varphi_{n-2}\right]$. The latter result can be found from the analysis of the first term in the low-energy-band Hamiltonian $\hat{H}_{2}$, which dominates for $|\epsilon|<\frac{1}{4} \gamma_{1}$ and high magnetic fields such that $\lambda_{B}^{-1}>\gamma_{1} v_{3} / v^{2}$. Numerical diagonalisation of the full $\hat{H}_{2}$ and $\hat{\mathcal{H}}_{2}$ shows ${ }^{\underline{5}}$ that this degeneracy is not lifted by the warping term $\delta \hat{H}_{w}$, and also the above-described grouping of LLs in bilayer graphene was confirmed in the recent QHE measurements ${ }^{3}$. It has been noticed 17 that degenerate levels with $n=0\left(\chi_{0, K_{+}}, \chi_{0, K_{-}}\right)$and $n=1\left(\chi_{1, K_{+}}, \chi_{1, K_{-}}\right)$can be weakly split by the second- 
neighbour hopping term $\beta p^{2} / m_{2}$, to $\varepsilon_{0}=\frac{\beta}{2} \hbar \omega_{c}$ and $\varepsilon_{1}=\frac{3 \beta}{2} \hbar \omega_{c}$, where $\beta \ll 1 \frac{18}{18}$. However, it is more likely that, at a high magnetic field, electrons in an ideally clean bilayer with filling factor $-4<\nu<4$ would form a correlated ground state in which the occupancy of $n=0$ and $n=1$ LLs would be determined by the electron-electron interaction. The correlated ground state may be particularly interesting in a bilayer with $\nu=0$. One can envisage $2 \mathrm{D}$ electrons forming a ferromagnetic QHE state in which both $n=0$ and $n=1$ states are half-filled (like in a convensional QHE liquid at the filling factors corresponding to a half-filled spin-degenerate LL). Alternatively, there can be an antiferromagnetic state with one fully occupied and one empty LL. Below we show that these two ground states can be distinguished experimentally on the basis of magneto-absorption spectra measured in circularly polarised FIR light.

Figure 2(a) illustrates the selection rules for the singleparticle inter-LL transitions in bilayer and monolayer graphene. In both cases, photons with in-plane $\frac{19}{}$ polarisation $\ell_{\ominus}$ are absorbed via transitions where the LL index changes from $n$ to $n-1(n \geq 1)$, whereas absorption of $\boldsymbol{\ell}_{\oplus}$ photons happens via transitions from the LL $n$ to $n+1(n \geq 0)$. Assuming the same broadening $\hbar \tau^{-1}$ of all LLs $\frac{21}{2}$, we arrive at the following magnetoabsorption spectra for monolayer 15 and bilayer graphene (for $\epsilon_{\mathrm{L}}<\hbar \omega<\frac{1}{4} \gamma_{1}$ ):

$$
\begin{gathered}
g_{J}^{\oplus / \ominus}(B, \omega)=\frac{2 \pi e^{2}}{\hbar c} f_{J}^{\oplus / \ominus}(B, \omega), \\
f_{1}^{\ominus}=\sum_{\substack{n \geq 1 \\
\alpha \alpha^{\prime}}} \frac{\frac{w_{1} \tau}{\pi \hbar} \frac{2 b_{n-1}^{2}}{\alpha \sqrt{n-1}-\alpha^{\prime} \sqrt{n}}\left(\nu_{n, \alpha^{\prime}}-\nu_{n-1, \alpha}\right)}{{\frac{\hbar^{2}}{2}}^{2}\left(\frac{\hbar \omega}{w_{1}}-\alpha \sqrt{n-1}+\alpha^{\prime} \sqrt{n}\right)^{2}+1} \\
f_{1}^{\oplus}=\sum_{\substack{n \geq 0 \\
\alpha \alpha^{\prime}}} \frac{\frac{w_{1} \tau}{\pi \hbar} \frac{2 b_{n}^{2}}{\alpha \sqrt{n+1}-\alpha^{\prime} \sqrt{n}}\left(\nu_{n, \alpha^{\prime}}-\nu_{n+1, \alpha}\right)}{\hbar^{2}}\left(\frac{\hbar \omega}{w_{1}}-\alpha \sqrt{n+1}+\alpha^{\prime} \sqrt{n}\right)^{2}+1 \\
f_{2}^{\ominus}=\sum_{n \geq 2} \frac{\frac{4 c_{n-1}^{2}}{\pi \omega_{c} \tau} \frac{\left(\nu_{n, \alpha^{\prime}}-\nu_{n-1, \alpha}\right)(n-1)}{\alpha \sqrt{n^{2}-n}-\alpha^{\prime} \sqrt{(n-1)(n-2)}}}{\left[\frac{\omega}{\omega_{c}}-\alpha \sqrt{n^{2}-n}+\alpha^{\prime} \sqrt{(n-1)(n-2)}\right]^{2}+\frac{\tau^{-2}}{\omega_{c}^{2}}} \\
f_{2}^{\oplus}=\sum_{\substack{n \geq 1 \\
\alpha \alpha^{\prime}}} \frac{\frac{4 c_{n}^{2}}{\pi \omega_{c} \tau} \frac{\left(\nu_{n, \alpha^{\prime}}-\nu_{n+1, \alpha}\right) n}{\alpha \sqrt{n^{2}+n}-\alpha^{\prime} \sqrt{n^{2}-n}}}{\left(\frac{\omega}{\omega_{c}}-\alpha \sqrt{n^{2}+n}+\alpha^{\prime} \sqrt{n^{2}-n}\right)^{2}+\frac{\tau^{-2}}{\omega_{c}^{2}}} .
\end{gathered}
$$

Here $w_{1}=\sqrt{2} \hbar v \lambda_{B}^{-1}$ and $\hbar \omega_{c}=\hbar^{2} / m_{2} \lambda_{B}^{2}$ are the characteristic energy scales for the LL spectra in monolayer $\left(J=1 ; \epsilon_{n \alpha}\right)$ and bilayer $\left(J=2 ; \varepsilon_{n \alpha}\right)$ graphene, respectively, and $\alpha, \alpha^{\prime}= \pm$ determine whether the corresponding state belongs to the conduction $(+)$ or valence $(-)$ band. Also, $\nu_{n, \alpha}$ are the filling factors of the corresponding LLs, and $b_{0}=1, b_{n \geq 1}=1 / \sqrt{2}$ for a monolayer and $c_{0,1}=1, c_{n \geq 2}=1 / \sqrt{2}$ for the bilayer.

One can test the selection rules shown in Fig. 2(a) and the actual polarisation of these transitions using gated graphene structures ${ }^{1,2,3}$. By filling the monolayer sheet
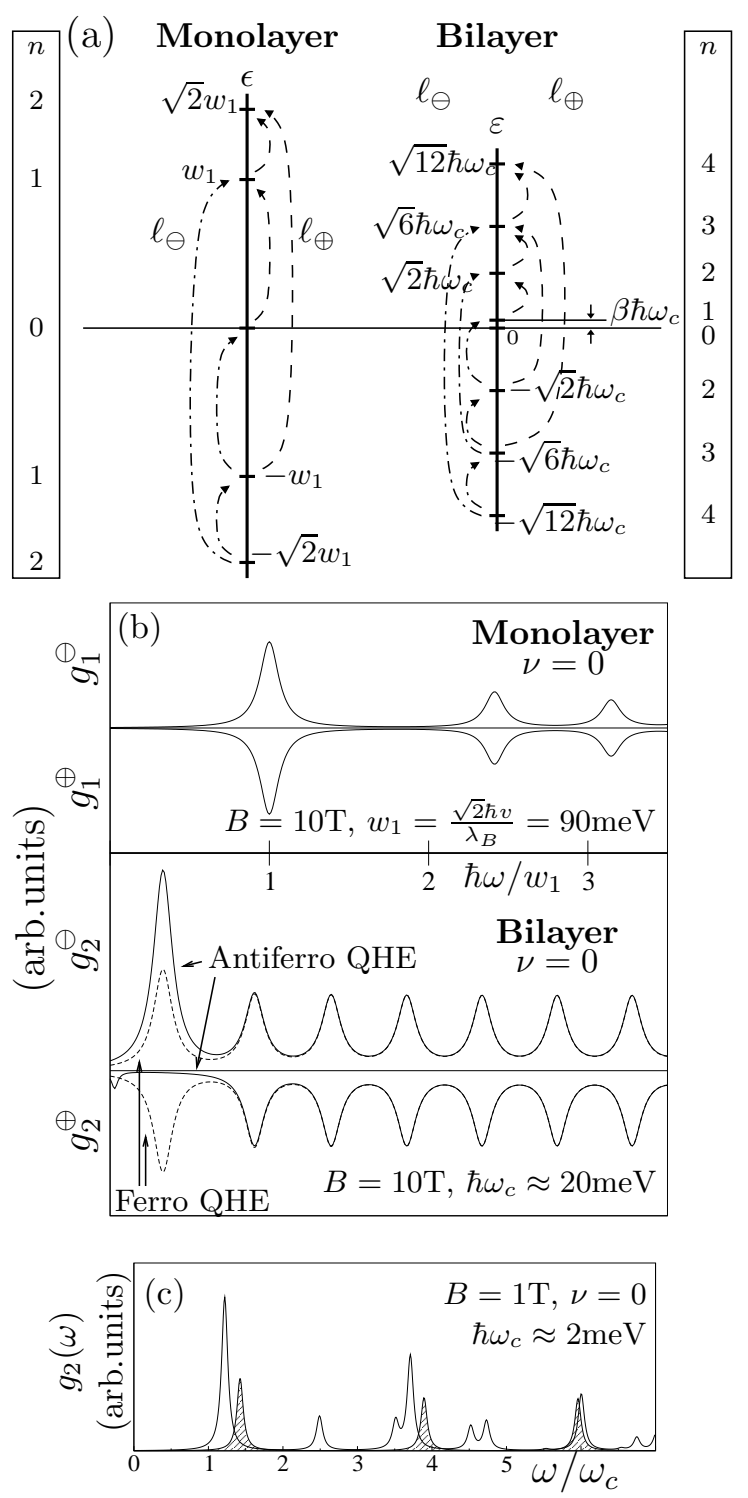

FIG. 2: (a) Allowed inter-LL transitions without trigonal warping effects. Dashed and dash-dot lines indicate transitions in $\ell_{\oplus}$ and $\ell_{\ominus}$ polarisations respectively. (b) Monolayer (top) and bilayer (bottom) FIR absorption spectra in $\ell_{\oplus}$ and $\ell_{\ominus}$ polarisations for $B=10 T$ and filling factor $\nu=0$. Dashed and solid lines describe absorption by ferro- and antiferromagnetic states of the $\nu=0$ bilayer. (c) Weak field, $B=1 \mathrm{~T}$ magnetoabsorption in bilayer graphene with $\nu=0$ calculated with $\left(v_{3}=0.2 v\right)$ and without $\left(v_{3}=0\right.$, shaded $)$ warping term in the Hamiltonian.

with electrons up to $\nu=2$ (a completely filled $n=0$ LL), one would suppress the intensity of the lowest absorption peak in $\boldsymbol{\ell}_{\ominus}$ polarisation and increase the size of the $\boldsymbol{\ell}_{\oplus}$ peak. By depleting the monolayer to $\nu=-2$ state (emptying the $n=0 \mathrm{LL}$ ) one would achieve the opposite effect. Similarly, in a bilayer with a completely filled pair of $n=0,1$ LLs which takes place at $\nu=4$, light with $\omega=\sqrt{2} \omega_{c}$ can be absorbed only in the $\boldsymbol{\ell}_{\oplus}$ polarisation. By depleting the bilayer down to $\nu=-4$ one could sup- 
press the absorption in this line in $\boldsymbol{\ell}_{\oplus}$ polarisation while retaining $\ell_{\ominus}$ absorption.

The denser magneto-absorption spectrum in bilayer graphene (as compared to the monolayer) is one of its features illustrated in Fig,2 (b). Another feature is that the intensity of the lowest absorption peak measured in the $\boldsymbol{\ell}_{\oplus}$ or $\boldsymbol{\ell}_{\ominus}$ polarisations at the filling factor $\nu=0$ may differ for different ground states of the bilayer. To illustrate this possibility, let us compare the absorption spectra for two model ground states of the bilayer.

The first can be attributed to a bilayer with such a large single-particle splitting between the $n=0$ and $n=1$ states that one of these levels is full and the other empty, even in the presence of the electron-electron interaction. This may be due either to the inter-layer assymmetry ${ }^{5,12}$ or caused by the AA/BB intralayer hopping in the last term of $\hat{H}_{2}$, Equation (5). Below we refer to such a QHE state as being antiferromagnetic, stressing that this state is not spin-polarised.

An alternative form of the ground state can be attributed to the case of a negligible splitting between the $n=0$ and $n=1$ LLs. In the latter situation the electronelectron repulsion may lead to the ferromagnetic alignment of electron spins and the formation a ferromagnetic QHE state with one spin component of each LL being completely full and the other completely empty.

Since transitions from/to the $n=0 \mathrm{LL}$ with $\varepsilon_{0} \approx 0$ to/from the states $\varepsilon_{n \pm}$ with $n \geq 2$ are forbidden, the intensity and polarisation of the peaks at $\omega=\sqrt{2} \omega_{c}$ are determined by transitions from/to $n=1 \mathrm{LL}$ (also with $\left.\varepsilon_{1} \approx 0\right)$ and directly reflect the occupancy of this state. If the bilayer ground state is ferromagnetic, with a halffilled $n=1 \mathrm{LL}$, the absorption peak at $\omega=\sqrt{2} \omega_{c}$ will have the same intensity in both $\boldsymbol{\ell}_{\oplus}$ and $\boldsymbol{\ell}_{\ominus}$ polarisations.

In contrast, absorption by a $\nu=0$ bilayer with antiferromagnetic ground state would contain the line at $\omega=\sqrt{2} \omega_{c}$ only in one polarisation: in $\ell_{\ominus}$ if $n=1 \mathrm{LL}$ is empty (fully occupied $n=0 \mathrm{LL}$ ) and in $\ell_{\oplus}$ if $n=1$ LL is full. For comparison, the lowest peak in the spectrum of a monolayer with $\nu=0$ appears in both polarisations, since both transitions to $\left(\epsilon_{1-} \rightarrow \epsilon_{0}\right)$ and from $\left(\epsilon_{0} \rightarrow \epsilon_{1+}\right)$ half-filled $n=0$ monolayer LL are possible All higher-energy absorption peaks which involve transitions between filled valence band states $(\alpha=-)$ and empty states in the conduction band $(\alpha=+)$ have equal strengths in both polarisations, which reflect effectively the inter-band nature of these transitions. Also, note that a weak transition between $n=0$ and 1 LLs with a low frequency $\beta \omega_{c}$ (microwave range) is possible, to the measure of a small $\beta$ and depending on the LL filling.

We briefly turn our attention to the effect of the electron-electron interaction on the absorption. Since we have specified that we study the spectrum for exactly filled levels we can construct magneto-exciton operators for each case $\mathrm{e}^{22}$. These operators correspond to collective excitations of electrons from fully filled to completely empty LLs. We calculated the shift in the dispersion of such magneoexcitons caused by these interactions to the first order in the interaction constant $e^{2} /\left(\epsilon_{r} \lambda_{B} \hbar \omega_{c}\right)$ - where $\epsilon_{r}$ is the dielectric constant of graphene - and find that the Coulomb interaction has no effect on these excitations when their in-plane wave vector $\mathbf{k}$ is zero. This result agrees with recent studies of the mangetoexciton dispersion in the integer QHE states in monolayer graphene ${ }^{23}$ and our previous work in the context of semiconductor heterostructures probed with surface acoustic waves $^{24}$. Therefore in these cases we can disregard the electron-electron interactions and say that our conclusions concerning the polarisation properties of FIR absorption apply to all magnetoexcitons which are symmetric in the spin and valley indices.

The analysis mentioned above was performed in the lowest order of pertubation theory in the interaction. Its result does not imply a full Kohn's theorem as known for electron-electron interactions in a simple parabolic band. In graphene, the chirality of the carriers is similar to the spin-orbit coupling in III-V semiconductors in that it plays the role of a non-parabolicity in the band structure which is known to violate Kohn's theorem in conventional semiconductors. This means that the conclusions for the lowest order corrections due to the interaction may not stand for higher orders of pertubation theory. This issue will be the subject of a seperate investigation ${ }^{25}$.

Finally, the warping term $\delta \hat{h}_{w}$ in Eq. (5) mixes each state $\chi_{n \alpha, K}$ with states $\chi_{(n \pm 3) \alpha, K}$. This generates weak transitions (with the coefficient $\left.\delta g_{2} \sim\left(v_{3} m_{2} \lambda_{B} / \hbar\right)^{2} g_{2}\right)$ between states which are 2 and 4 levels apart. Although such transitions are negligibly weak at high fields where the first term in $\hat{H}_{2}$ is dominant, they become relevant at weak fields, where $\lambda_{B}^{-1} \lesssim \gamma_{1} v_{3} / v^{2}$, and the low-frequency absorption spectrum of a bilayer aquires an additional structure. In Fig 2(c) we compare the absorption spectra in a $\nu=0$ bilayer $^{21}$ at $B=1 \mathrm{~T}$ calculated numerically for $v_{3}=0$ and $v_{3}=0.2 v$.

In conclusion, we have described peculiar optical and FIR magneto-optical properties of bilayer graphene and compared them with those of the monolayer material. The zero field optical absorption spectrum shown in Fig. 1 reflects the existance of four bands in the electronic spectrum of this material whereas the FIR magnetooptical spectrum of bilayer graphene, which is much denser than that of a monolayer, reflects the parabolic dispersion of its low-energy bands $\varepsilon_{c}^{ \pm}$. Due to the interband character of the inter-LL transitions in graphene with low carrier density, the magneto-optical spectrum in a monolayer and the higher energy part of the bilayer spectrum $\left(\omega>3 \omega_{c}\right)$ do not depend on the FIR light polarisation. However, the absorption peak at $\omega=\sqrt{2} \omega_{c}$ may appear differently in $\boldsymbol{\ell}_{\oplus}$ and $\boldsymbol{\ell}_{\ominus}$ polarised light, depending on the occupancy of the two zero-energy LL $(n=0$ and 1$)$ in bilayer graphene ${ }^{3,5}$. In such spectroscopic studies, the weakness of $g_{2}$ can be overcome once one uses the $2 \mathrm{D}$ electrons in graphene in the QHE regime for the transport detection of FIR absorption.

We thank E. McCann and A. Varlamov for discussions and the EPSRC grant EP/C511743 for support. This 
work has been complete during the MPI-PKS workshop 'Dynamics and Relaxation in Complex Quantum and
Classical Systems and Nanostructures', July-October 2006.
1 K. Novoselov, A. K. Geim, S. V. Morozov, D. Jiang, M. I. Katsnelson, I. V. Grigorieva, S. V. Dubonos and A. A. Firsov, Nature 438, 197 (2005); Science 306, 666 (2004)

2 Y. Zhang, J. P. Small, M. E. S. Amori and P. Kim, Phys. Rev. Lett. 94, 176803 (2005); Nature 438, 201 (2005)

3 K. Novoselov, K. S. Novoselov, E. McCann, S. V. Morozov, V.I. Fal'ko, M. I. Katsnelson, U. Zeitler, D. Jiang, F. Schedin and A. K. Geim, Nature Physics 2, 177 (2006)

${ }^{4}$ M. S. Dresselhaus and G. Dresselhaus, Adv. Phys. 51, 1 (2002); T. Ando, J. Phys. Soc. Jpn. 74, 777 (2005)

5 E. McCann and V.I. Fal'ko, Phys Rev. Lett. 96086805 (2006)

6 Y. Zheng, T. Ando, Phys. Rev. B 65, 245420 (2002); V. Gusynin, S. Sharapov, Phys. Rev. Lett. 95, 146801 (2005); A. Castro Neto, F. Guinea, N. Peres, Phys. Rev. B 73, 205408 (2006)

7 V. Gusynin, S. Sharapov, J. Carbotte, Phys. Rev. Lett. 96, 256802 (2006); V. Gusynin and S. Sharapov, Phys. Rev. B $73,245411(2006)$

8 J. Nilsson, A.H. Castro Neto, F. Guinea and N.M.R. Peres, Phys. Rev. Lett. 97, 266801 (2006).

${ }^{9}$ L. Falkovsky and A. Varlamov, cond-mat/0606800

10 J. Cserti, Phys. Rev. B 75, 033405 (2007)

11 K. Nomura and A.H. MacDonald, Phys. Rev. Lett. 96, 256602 (2006); T. Ando, J. Phys. Soc. Jpn. 75, 074716 (2006); V. Cheianov and V.I. Fal'ko, Phys. Rev. Lett. 97, 226801 (2006)

12 C. L. Lu, C. P. Chang, Y. C. Huang, R. B. Chen, and M. L. Lin, Phys. Rev. B 73, 144427 (2006); Johan Nilsson, A. H. Castro Neto, N. M. R. Peres, and F. Guinea, Phys. Rev. B 73, 214418 (2006); M. Koshino, T. Ando, Phys. Rev. B 73, 245403 (2006); F. Guinea, A. Castro Neto, N. Peres, Phys. Rev. B 73, 245426 (2006); B. Partoens, F. Peeters, Phys. Rev. B 74, 075404 (2006); S. Latil and L. Henrard, Phys. Rev. Lett. 97, 036803 (2006).

13 T. Ohta, A. Bostwick, T. Seyller, K. Horn, and E. Rotenberg, Science 313, 951 (2006)

14 J.W. McClure, Phys. Rev. 104, 666 (1956)

15 M. L. Sadowski, G. Martinez, M. Potemski, C. Berger, and
W. A. de Heer, Phys. Rev. Lett, 97, 266405 (2006);

16 Here, $\mathbf{K}_{ \pm}= \pm\left(\frac{2}{3} h a^{-1}, 0\right), a$ is the lattice constant.

17 E. McCann, Phys. Rev. B 74, 161403 (2006)

18 The sign of $\beta$ is not known, though one can estimate using bulk graphite parameters 4 that $|\beta| \lesssim 10^{-2}$.

19 In contrast to monolayer graphene, a weak absorption of light polarised perpendicular to the bilayer is possible. A pertubation $\sigma_{z} e E_{z} d / 2$ distinguishes between the on-site energies in the top and bottom layers separated by spacing $d$, which leads to weak absorption $g_{2}^{z}=\left(2 \pi e^{2} / \hbar c\right) f_{2}^{z}$,

$$
\begin{gathered}
f_{2}^{z}=a_{z}^{2} \Omega\left[\frac{1}{\Omega+1}+\frac{\theta(\Omega-2)}{\Omega-1}\right], \quad \Omega \equiv \hbar \omega / \gamma_{1} ; \\
f_{2}^{z}(B, \omega)=\frac{a_{z}^{2}}{\pi} \sum_{n \geq 2} \frac{\tau \omega}{\tau^{2} \omega_{c}^{2}\left(\frac{\omega}{\omega_{c}}-2 \sqrt{n^{2}-n}\right)^{2}+1}
\end{gathered}
$$

where the constant $a_{z}=\gamma_{1} d / 2 \hbar v \sim 10^{-1}$, and the magneto-absorption spectrum at $\hbar \omega<\frac{1}{4} \gamma_{1}$ involves $\varepsilon_{n-} \rightarrow$ $\varepsilon_{n+}$ inter-LL transitions.

20 For $\hbar \omega \ll \frac{1}{4} \gamma_{1}$ this result transforms into $f_{2}=1$ suggested by J.Cserti ${ }^{10}$ for the microwave absorption in bilayer graphene. However one should be aware that Eq. (7) and conclusions of Ref ${ }^{10}$ cannot be applied to $\hbar \omega \lesssim \epsilon_{\mathrm{L}}=$ $\frac{1}{2} \gamma_{1}\left(v_{3} / v\right)^{2} \sim 1 \mathrm{meV}$. At $\epsilon_{\mathrm{F}} \approx \epsilon_{\mathrm{L}}$, the warping term $\delta \hat{H}_{w}$ causes the Lifshitz transition ${ }^{26}$ in the topology of the Fermi line (in each valley): from singly-connected at $\epsilon_{\mathrm{F}}>\epsilon_{\mathrm{L}}$ to four pockets at $\epsilon_{\mathrm{F}}<\epsilon_{\mathrm{L}}$.

21 For energy-dependent broadening one replaces $\tau^{-1}$ in the sum in Eq. (8) with $\tau_{n \alpha, n^{\prime} \alpha^{\prime}}^{-1}=\frac{1}{2}\left(\tau_{n \alpha}^{-1}+\tau_{n^{\prime} \alpha^{\prime}}^{-1}\right)$.

22 C. Kallin and B. I. Halperin, Phys. Rev. B 30, 5655 (1984)

23 A. Iyengar, J. Wang, H. A. Fertig, and L. Brey, cond-mat/0608364

24 I. V. Kukushkin, J. H. Smet, D. S. Lyne Abergel, V. I. Fal'ko, W. Wegscheider, and K. von Klitzing, Phys. Rev. Lett. 96, 126807 (2006).

25 D. S. L. Abergel and V. I. Fal'ko, unpublished.

26 A.A. Abrikosov, Fundamentals of the Theory of Metals, North-Holland 1988 